Check for updates

Cite this: RSC Adv., 2018, 8, 29976

\title{
Characterization of the piezoresistance in highly doped p-type 3C-SiC at cryogenic temperatures $\uparrow$
}

Received 7th July 2018

Accepted 18th August 2018

DOI: $10.1039 / c 8 r a 05797 d$

rsc.li/rsc-advances

\author{
Hoang-Phuong Phan, (ID *ab Karen M. Dowling, ${ }^{b}$ Tuan-Khoa Nguyen, (DD ${ }^{a}$ \\ Caitlin A. Chapin, ${ }^{b}$ Toan Dinh, (D) ${ }^{a}$ Ruth A. Miller, ${ }^{b}$ Jisheng Han, ${ }^{a}$ Alan lacopi, \\ Debbie G. Senesky, ${ }^{\text {bc }}$ Dzung Viet Dao ad and Nam-Trung Nguyen (iD) ${ }^{a}$
}

\section{Introduction}

Wide band gap semiconductors such as gallium nitride (GaN), silicon carbide (SiC), and diamond like carbon (DLC) have been of significant interest for harsh environment applications. ${ }^{\mathbf{1 - 6}}$ Among these materials, SiC has proved to be an outstanding candidate owing to its chemical inertness, availability of materials, as well as well-established fabrication technology including the capability of growing on a Si substrate. Numerous SiC based Micro Electro Mechanical Systems (MEMS) sensors have been developed, including UV sensors, gas sensors, and mechanical sensors. ${ }^{7-12}$ For structural health monitoring systems, the use of mechanical sensors is imperative; for example, strain sensors can detect crack generation, while pressure sensors can be employed for the feedback control of an engine to enhance its fuel efficiency. A large number of studies have been carried out to investigate the piezoresistance in SiC for mechanical sensing applications. With the capability of tuning the energy bands under strain, SiC exhibits a relatively high gauge factor ranging from 10 to $50 .{ }^{13-16}$ Along with the large Young's modulus and excellent mechanical strength, the piezoresistive effect in SiC has been utilized in applications requiring fast response and extreme mechanical loads such as

${ }^{a}$ Queensland Micro-Nanotechnology Centre, Griffith University, Queensland, Australia. E-mail: hoangphuong.phan@griffithuni.edu.au

${ }^{b}$ Department of Aeronautics and Astronautic, Stanford University, USA

${ }^{c}$ Department of Electrical Engineering, Stanford University, CA, USA

${ }^{d}$ School of Engineering, Griffith University, Queensland, Australia

$\dagger$ Electronic supplementary information (ESI) available. See DOI: 10.1039/c8ra05797d high-shock sensors and high-g accelerometers. ${ }^{17}$ Additionally, piezoresistive SiC pressure sensors which can operate at high temperatures have also been demonstrated. ${ }^{18,19}$ For instance, Okojie et al. developed $4 \mathrm{H}-\mathrm{SiC}$ pressure sensors which can function up to $1000 \mathrm{~K} .{ }^{20}$ Although numerous studies have been conducted on SiC at high temperatures, the piezoresistance of the material at negative temperatures has rarely been reported. As low temperature tolerance is imperative in several fields (e.g. space exploration where temperatures can be below $100 \mathrm{~K}){ }^{21,22}$ investigation into the feasibility of SiC for low temperature applications is significant.

This work reports for the first time the piezoresistance of $\mathrm{p}$ type $3 \mathrm{C}$-SiC at low temperatures down to $150 \mathrm{~K}$. Our experimental results show that the gauge factor of $\mathrm{p}$-type 3C-SiC films slightly increased with decreasing temperature, which is in good agreement with theoretical analysis.

\section{Results and discussion}

The growth process of the SiC film can be found elsewhere, ${ }^{23}$ in which low pressure chemical vapor deposition was utilized to epitaxially deposit $\mathrm{SiC}$ on a 6-inch Si wafer (p-type with a carrier concentration of $10^{14} \mathrm{~cm}^{-3}$ ). The crystal quality and surface roughness of the SiC films were reported in our previous studies. ${ }^{24}$ The SiC films were found to be p-type semiconductor with the carrier concentration found to be approximately $2 \times$ $10^{19} \mathrm{~cm}^{-3}$ using Hall-measurement and/or hot probe technique. Following the SiC deposition process, lithography and inductive coupled plasma etching were employed to form SiC micro resistors with dimension of $550 \mu \mathrm{m} \times 50 \mu \mathrm{m} \times 380 \mathrm{~nm}$. 


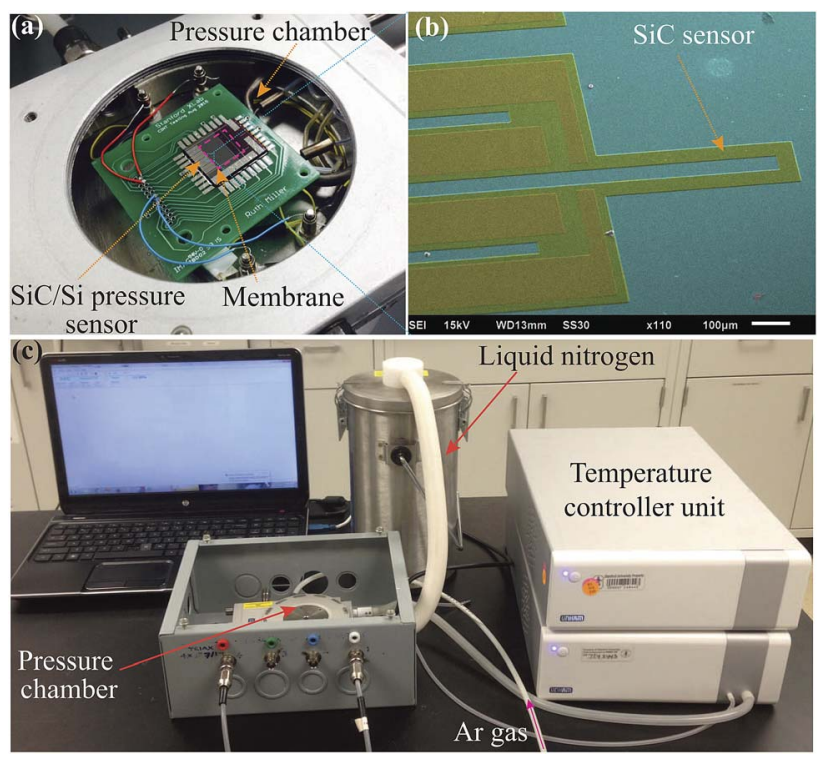

Fig. 1 Photographs of fabricated device and the experimental setup. (a) SiC on Si pressure sensor mounted on a PCB board; (b) SEM image of a $\mathrm{SiC}$ resistor (false colored); (c) experimental setup for characterization of the piezoresistive effect in $3 \mathrm{C}$-SiC at cryogenic temperature.

Subsequently, aluminum was deposited and patterned to form the electrodes for the SiC resistors. Finally, UV laser engraving was utilized to form $\mathrm{SiC}$ on $\mathrm{Si}$ diaphragm with dimensions of $7 \mathrm{~mm} \times 7 \mathrm{~mm} \times 150 \mu \mathrm{m}$. UV laser engraving was chosen due to the high etching rate of $16 \mu \mathrm{m} \mathrm{min}^{-1}$ in comparison to other dry etching methods with etching rates of typically below 10 $\mu \mathrm{m} \mathrm{min}{ }^{-1}$. This high etching rate allows quick prototyping of pressure sensors. ${ }^{25}$ In addition, the use of laser engraving eliminates the requirement for the hard-mask used in backsideSi-etching, which significantly simplifies our fabrication process (see the ESI $\dagger$ ). Subsequently, the as-fabricated pressure sensor was mounted on a home-built PCB board to form an enclosed chamber under the SiC/Si membrane. Fig. 1(a) and (b) show photographs of the $\mathrm{SiC} / \mathrm{Si}$ pressure sensor.

Initially, the piezoresistive effect of the SiC film was characterized at room temperature using the experimental setup shown in Fig. 1(c) where the PCB boards were placed on a heating/cooling chuck. A thin layer of thermal grease was applied between the surface of the PCB and the chuck to enhance the thermal conductivity. The Linkam ${ }^{\mathrm{TM}}$ chamber was then filled with Ar gas to apply pressure to the sensor. The applied pressure was controlled using a pressure regulator with a manual incrementation of approximately 0.25 bar per step. Further detail of the experimental setup and its schematic sketch are presented in the ESI. $\dagger$ The applied voltage at the SiC resistor was then fixed at $0.5 \mathrm{~V}$, and the current was monitored and recorded using an Agilent ${ }^{\mathrm{TM}}$ Semiconductor Device Analyzer B1500 while increasing the gas pressure. It is evident from Fig. 2(a) that the current decreased with increasing pressure from 0 to $2.1 \mathrm{bar}$, indicating a positive gauge factor in the $\mathrm{p}$ type 3C-SiC. The current also returned to its initial value when the applied pressure was completely released. Additionally, the variation in the current due to the applied pressure at room temperature shows excellent repeatability after several pressurizing/depressurizing cycles, as illustrated in Fig. 2(b).

Fig. 2(c) plots the resistance change of the SiC resistor against applied pressure, showing a good linearity which is preferable for mechanical sensing applications. The strain induced into the $\mathrm{SiC}$ resistors was estimated based on finite element analysis (FEA) using COMSOL Multiphysics ${ }^{\mathrm{TM}}$. It should be pointed out that since the thickness of the $\mathrm{Si}$ substrate was significantly larger than that of the Si membrane, the strain applied to the SiC piezoresistor was almost the same as that of the top surface of the Si membrane. Additionally, since the SiC resistor was located at the center of the edge of the $\mathrm{SiC} / \mathrm{Si}$ membrane, the shear and transverse strains were approximately two orders of magnitude smaller than that of longitudinal strain, Fig. 2(c) inset. Therefore, only the longitudinal gauge factor significantly contributed to the response of the SiC resistors under applied pressure, while the influence of shear and transverse gauge factors was negligible. Based on the simulation results, the longitudinal strain applied to the SiC resistor under a pressure of 2 bar was found to be approximately $600 \mathrm{ppm}$. Consequently, the longitudinal gauge factor $\left(\mathrm{GF}_{1}=\right.$ $(\Delta R / R) / \varepsilon)$ of the $3 \mathrm{C}$-SiC resistors were found to be 30 . This result was consistent with our previously reported results, measured using the bending beam method. ${ }^{16}$

The temperature of the device was then reduced using liquid nitrogen. The temperature of SiC/Si chip was estimated by the (a)

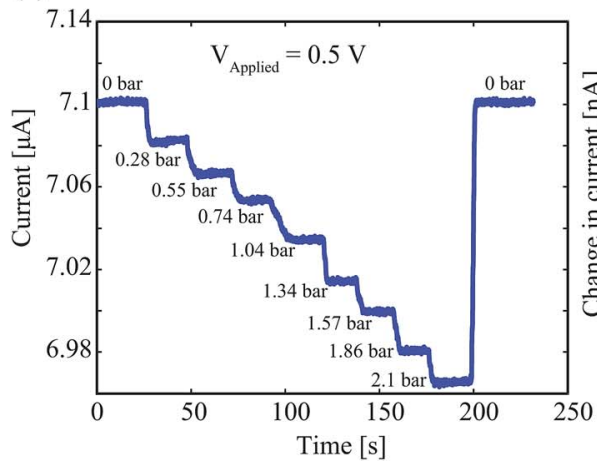

(b)

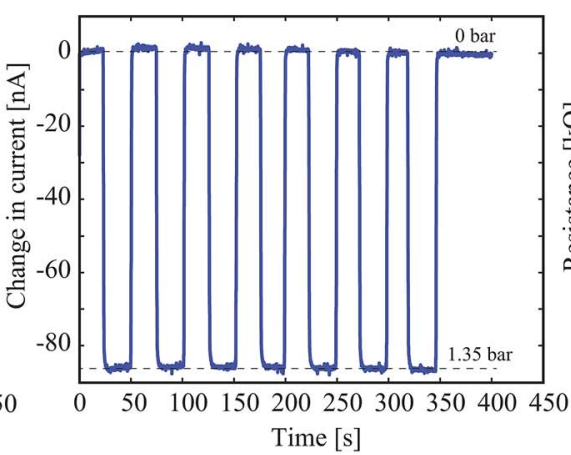

(c)

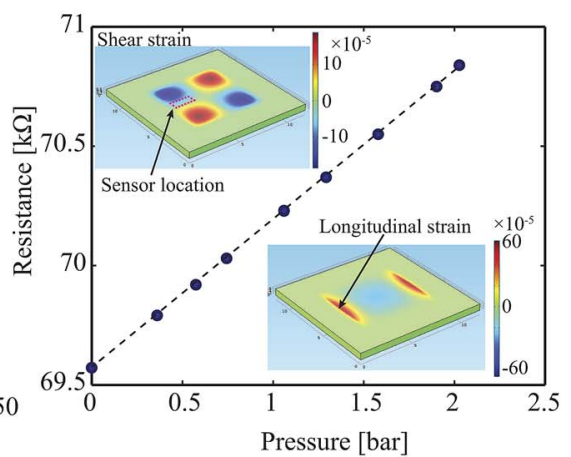

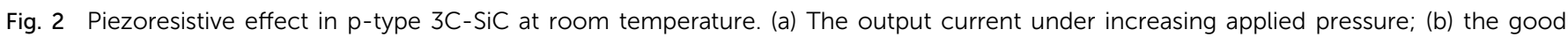

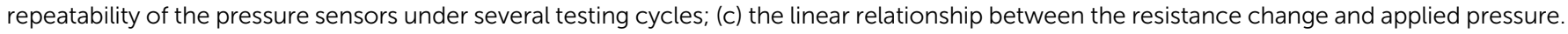




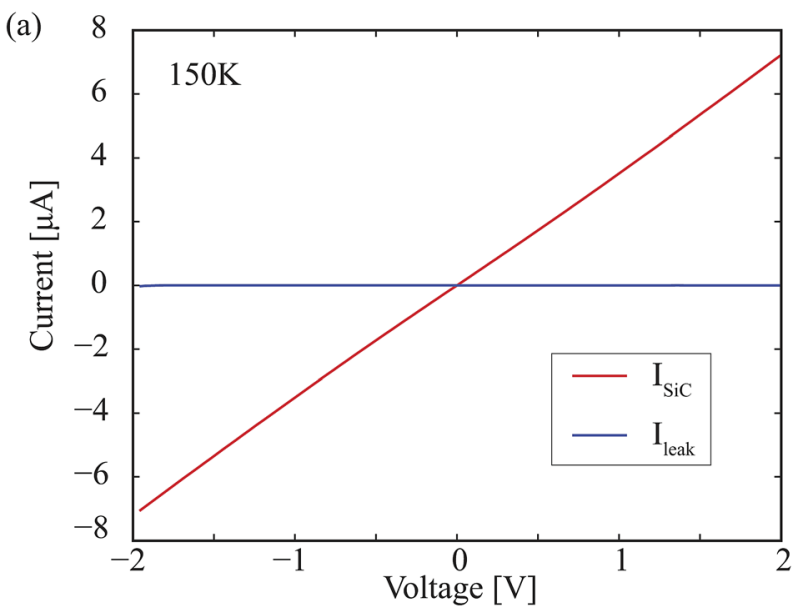

(b)

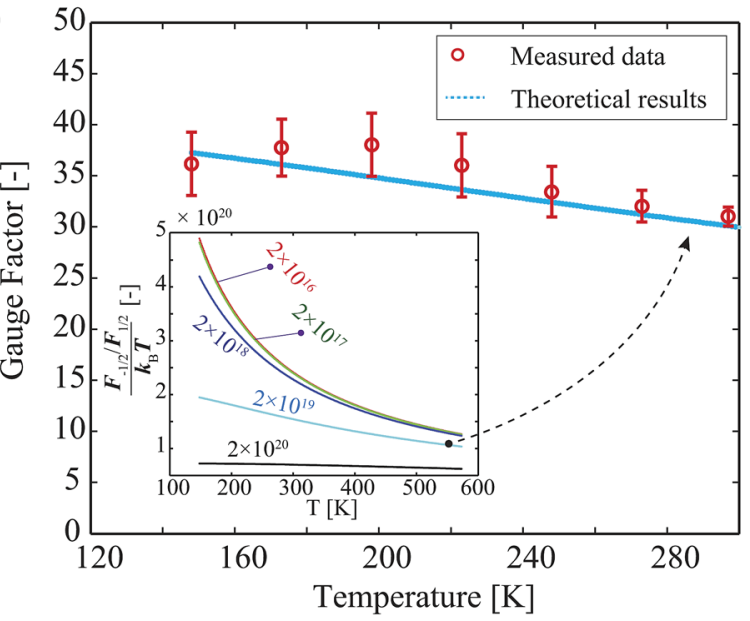

Fig. 3 Piezoresistive effect in p-type 3C-SiC at cryogenic temperature. (a) Good ohmic contact and small current leakage were maintained at low temperatures. (b) The gauge factor of $\mathrm{SiC}$ at room temperature down to $150 \mathrm{~K}$. Inset: estimation of the Fermi-Dirac integral $\left(\mathcal{F}_{-1 / 2} / \mathcal{F}_{1 / 2} / k_{\mathrm{B}} T\right)$ in a wide range of temperatures at different carrier concentration (unit: $\mathrm{cm}^{-3}$ ).

set-temperature of the thermal chuck after a calibration test relating the chuck temperature to the temperature reported by a commercial thermocouple epoxied to the surface was completed. The $I-V$ curve of the resistance at $150 \mathrm{~K}$ shows good ohmic contact between $\mathrm{Al}$ and p-type $\mathrm{SiC}$ was maintained at low temperatures. Furthermore, the current leakage to the substrate at low temperatures was significantly lower than the current passing through the SiC resistors as plotted in Fig. 3(a). This is due to the discontinuity between the valance bands of SiC and $\mathrm{Si}$ as well as the reduction of the charge carriers in Si substrate, resulting in less hole tunneling through the potential barrier between $\mathrm{SiC}$ and $\mathrm{Si}$ at low temperature. It should also be noted that, the designed $\mathrm{SiC}$ resistors possess four electrode pads, enabling four-terminal measurement. However, since the contact resistance was significantly smaller than that of the SiC resistor, the two-terminal measurement was applied to monitor the change of SiC resistance under applied pressure.

The piezoresistive effect of the SiC pressure sensors at cryogenic temperature was then investigated by pressurizing the chamber using Ar gas as described above. The gauge factor of the SiC films at low temperature was calculated in which the change in the stiffness of $\mathrm{Si}$ membrane with temperature was taken into account $\left(\Delta E_{\mathrm{Si}}(T)=E_{\mathrm{Si}} \times \Delta T \times \gamma\right.$, where $\gamma=-50 \mathrm{ppm}$ $\mathrm{K}^{-1}$ is the temperature coefficient of Young's modulus). ${ }^{26}$ Fig. 3(c) shows the relationship between the gauge factors and temperature. It is evident that, there was a small change in the piezoresistive effect in the p-type $3 \mathrm{C}$-SiC at low temperature; the gauge factor increased from 30 at $300 \mathrm{~K}$ to 36 at $150 \mathrm{~K}$. The piezoresistive effect in p-type semiconductors is caused by the splitting of heavy hole and light hole bands when the materials are subjected to a mechanical strain. Under the deformation of the energy structures, the holes will re-populate between these two bands, following the Boltzmann distribution: ${ }^{27}$

$$
\Delta p(i)=-\frac{N_{\mathrm{v}(i)} \Delta\left(E_{\mathrm{v}(i)}-E_{\mathrm{F}}\right)}{k_{\mathrm{B}} T} \mathcal{F}_{-1 / 2}
$$

where $i=1,2$ denotes the heavy hole and light hole; $N_{\mathrm{v}}$ is the effective density of state in the valence band; $E_{\mathrm{F}}$ is the Fermi level; $k_{\mathrm{B}}$ is the Boltzmann constant; and $\mathcal{F}$ is the Fermi-Dirac integral, respectively. Assuming that the total number of holes in highly doped semiconductors remains constant under applied strain $\left(\right.$ i.e. $\left.\sum_{i=1}^{2} \Delta p(i)=0\right),{ }^{28}$ the changing rate of the charge carrier in the heavy and light hole bands is given by: ${ }^{27}$

$$
\frac{\Delta p(i)}{p}=\frac{\Delta E_{\mathrm{v}(i)} \sum_{i=1}^{2} N_{\mathrm{v}(i)}-\sum_{i=1}^{2} \Delta E_{\mathrm{v}(i)} N_{\mathrm{v}(i)}}{\left(\sum_{i=1}^{2} N_{\mathrm{v}(i)}\right)^{2}} \frac{\mathcal{F}_{-1 / 2} / \mathcal{F}_{1 / 2}}{k_{\mathrm{B}} T}
$$

Since the change in the electrical conductance is propositional to the modification of the hole concentration, the gauge factor of p-type semiconductor can be simplified as: ${ }^{29}$

$$
\mathrm{GF} \approx C \times \frac{1}{k_{\mathrm{B}} T} \times \frac{\mathcal{F}_{-1 / 2}}{\mathcal{F}_{1 / 2}}
$$

where $C$ is a constant representing the magnitude of the piezoresistive effect, which can be empirically obtained from the gauge factor measured at room temperature. Accordingly, the third component of eqn (3) $\left(\left(\mathcal{F}_{-1 / 2} / \mathcal{F}_{1 / 2}\right) / k_{\mathrm{B}} T\right)$ depends not only on temperature but also on the carrier concentration. This component was calculated using the Chang-Izabelle ${ }^{30}$ approximating method and plotted in the inset of Fig. 3. The analytical result show that $\mathcal{F}_{-1 / 2} / \mathcal{F}_{1 / 2} / k_{\mathrm{B}} T$ was higher at low carrier concentrations, exhibiting the same trend as experimental data reported in the literature. ${ }^{31}$ It also evident that the ratio of the Fermi-Dirac integral to $k_{\mathrm{B}} T$ was relatively stable for high carrier concentration, but changes markedly with temperature for low doped SiC. This result was consistent with our experimental data plotted in Fig. 3(b) showing the stability of the piezoresistive effect in p-type 3C-SiC at temperatures down to $150 \mathrm{~K}$.

\section{Conclusion}

This work investigated the piezoresistive effect in highly doped p-type 3C-SiC grown on a $\mathrm{Si}$ substrate in cryogenic 
temperatures. A large and temperature-stable gauge factor of above 30 was found in the p-type 3C-SiC. The stability and the high sensitivity of the piezoresistive effect demonstrate the potential of p-type $3 \mathrm{C}$-SiC for mechanical sensing at cryogenic temperatures.

\section{Conflicts of interest}

There are no conflicts to declare.

\section{Acknowledgements}

This work was partially funded by the linkage grants LP150100153 and LP160101553 from the Australian Research Council (ARC). This work was performed in part at the Queensland node of the Australian National Fabrication Facility, a company established under the National Collaborative Research Infrastructure Strategy to provide nano and microfabrication facilities for Australia's researchers. H.-P. Phan acknowledges research grants from the Australian Nanotechnology Network Overseas Travel Fellowship and the Griffith University New Researcher Grant.

\section{References}

1 D. G. Senesky, B. Jamshidi, K. B. Cheng and A. P. Pisano, IEEE Sens. J., 2009, 9, 1472-1478.

2 A. Qamar, P. Tanner, D. V. Dao, H. P. Phan and T. Dinh, IEEE Electron Device Lett., 2014, 35(12), 1293-1295.

3 F. Gao, J. Zheng, M. Wang, G. Wei and W. Yang, Chem. Commun., 2011, 47(43), 11993-11995.

4 A. L. Vikharev, A. M. Gorbachev, M. A. Lobaev, A. B. Muchnikov, D. B. Radishev, V. A. Isaev, V. V. Chernov, S. A. Bogdanov, M. N. Drozdov and J. E. Butler, Phys. Status Solidi RRL, 2016, 10(4), 324-327.

5 T. Chen, Y. Huang, D. Yang, R. Carius and F. Finger, Phys. Status Solidi RRL, 2010, 4(34), 61-63.

6 H. P. Phan, H. H. Cheng, T. Dinh, B. Wood, T. K. Nguyen, et al., ACS Appl. Mater. Interfaces, 2017, 9(33), 27365-27371.

7 M. Massimo, P. Shukla, R. Mallik, M. Kumar, R. Previti, G. D. Marco, A. Sciuto, R. A. Puglisi and V. Raineri, IEEE Sens. J., 2011, 11(2), 377-381.

8 K.-S. Kim and G.-S. Chung, Sens. Actuators, B, 2011, 160(1), 1232-1236.

9 H. P. Phan, T. Dinh, T. Kozeki, T. K. Nguyen, A. Qamar, T. Namazu, N. T. Nguyen and D. V. Dao, Appl. Phys. Lett., 2016, 109(12), 123502.

10 T. Dinh, H. P. Phan, A. Qamar, P. Woodfield, N. T. Nguyen and D. V. Dao, J. Microelectromech. Syst., 2017, 26(5), 966986.
11 J. Bi, G. Wei, L. Wang, F. Gao, J. Zheng, B. Tang and W. Yang, J. Mater. Chem. C, 2013, 1(30), 4514-4517.

12 X. Li, S. Chen, P. Ying, F. Gao, Q. Liu, M. Shang and W. Yang, J. Mater. Chem. C, 2016, 4(27), 6466-6472.

13 T. K. Nguyen, H. P. Phan, T. Dinh, J. Han, S. Dimitrijev, P. Tanner, A. R. M. Foisal, Y. Zhu, N. T. Nguyen and D. V. Dao, Electron Device Lett., 2017, 38(7), 955-958.

14 M. A. Fraga, H. Furlan, R. S. Pessoa, L. A. Rasia and C. F. R. Mateus, Microsyst. Technol., 2012, 18(7-8), 10271033.

15 R. Shao, K. Zheng, Y. Zhang, Y. Li, Z. Zhang and X. Han, Appl. Phys. Lett., 2012, 101(23), 233109.

16 H. P. Phan, T. Dinh, T. Kozeki, T. K. Nguyen, A. Qamar, T. Namazu, N. T. Nguyen and D. V. Dao, Electron Device Lett., 2016, 37(8), 1029-1032.

17 A. R. Atwell, R. S. Okojie, K. T. Kornegay, S. L. Roberson and A. Beliveau, Sens. Actuators, A, 2003, 104(1), 11-18.

18 R. S. Okojie, C. Blaha, D. Lukco, V. Nguyen and E. Savrun, 2010 IEEE Sensors Conf., 2010, pp. 2269-2274.

19 C. H. Wu, C. A. Zorman and M. Mehregany, IEEE Sens. J., 2006, 6(2), 316-324.

20 R. S. Okojie, D. Lukco, V. Nguyen and E. Savrun, Electron Device Lett., 2015, 36(2), 174-176.

21 H. So and D. G. Senesky, IEEE Sens. J., 2017, 17(15), 47524756.

22 S. J. Zhang, F. Li and F. P. Yu, in Structural Health Monitoring (SHM) in Aerospace Structures, 2016, pp. 59-93.

23 L. Wang, S. Dimitrijev, J. Han, et al., J. Cryst. Growth, 2011, 329, 67-70.

24 A. Qamar, H. P. Phan, J. Han, P. Tanner, T. Dinh, L. Wang, S. Dimitrijev and D. V. Dao, J. Mater. Chem. C, 2015, 3(34), 8804-8809.

25 K. M. Dowling, H. So, A. Toor, C. A. Chapin and D. G. Senesky, Microelectron. Eng., 2017, 173, 54-57.

26 E. J. Boyd, L. Li, R. Blue and D. Uttamchandani, Sens. Actuators, A, 2013, 198, 75-80.

27 T. Toriyama and S. Sugiyama, J. Microelectromech. Syst., 2002, 11(5), 598-604.

28 Y. Sun, S. E. Thompson and T. Nishida, Strain effect in semiconductors: theory and device applications. Springer, 2009.

29 H. P. Phan, D. V. Dao, K. Nakamura, S. Dimitrijev and N. T. Nguyen, J. Microelectromech. Syst., 2015, 24(6), 16631677.

30 T. Y. Chang and A. Izabelle, J. Appl. Phys., 1989, 65(5), 21622164.

31 M. Eickhoff, M. Moller, G. Kroetz, et al., J. Appl. Phys., 2004, 96, 2872-2879. 